\title{
Análisis Retrospectivo de los Tratamientos Quirúrgicos en el área de Cirugía Oral y Maxilofacial en el Hospital Metropolitano. Periodo 2015-2018
}

Retrospective Analysis of Surgical Treatments in the area of Oral and Maxillofacial Surgery at the Metropolitan Hospital. 2015-2018 period

\section{Evelyn Olalla L. ${ }^{1}$ Fernando J. Sandoval P. ${ }^{2}$ Fernando Sandoval V. ${ }^{3}$}

\author{
Universidad San Francisco de Quito, Colegio de Ciencias de la Salud, Escuela de \\ Odontología, Clínica Odontológica, Campus Cumbayá, Oficina C0 106, casilla postal \\ 17-1200-841. Quito-Ecuador. Correo electrónico: evelynolalla@hotmail.com \\ 2Universidad San Francisco de Quito, Colegio de Ciencias de la Salud, Escuela de \\ Odontología, Clínica Odontológica, Campus Cumbayá, Oficina C0 106, casilla postal \\ 17-1200-841. Quito-Ecuador. Correo electrónico: fjose18@hotmail.com \\ ${ }^{3}$ Universidad San Francisco de Quito, Colegio de Ciencias de la Salud, Escuela de \\ Odontología, Clínica Odontológica, Campus Cumbayá, Oficina C0 106, casilla postal \\ 17-1200-841. Quito-Ecuador. Correo electrónico: fsandoval@usfa.edu.ec
}

Editado por / Edited by: Johanna Monar

Recibido / Received: 06-08-2019

Aceptado / Accepted: 24-09-2019

Publicado en linea / Published online: 30-09-2019 


\section{Resumen}

La cirugía oral y maxilofacial es una especialidad medico quirúrgica, que se ocupa del diagnóstico y tratamiento de traumatismos faciales, anomalías dentofaciales, fisuras labio palatinas, infecciones de origen odontogénico, procesos patológicos, oncológicos o neoplásicos que involucran la cavidad bucal, cara y territorio craneofacial, así como de los órganos y estructuras cervicales relacionadas directa o indirectamente con las mismas y que requieren un tratamiento conservador o quirúrgico. El objetivo de este estudio fue determinar la etiología tratamientos quirúrgicos que se realizaron en el Hospital Metropolitano y su prevalencia durante el periodo 2015-2018. Se realizó un estudio de tipo descriptivo, cuantitativo, retrospectivo y transversal. La muestra de la investigación fue de 245 pacientes que fueron atendidos e intervenidos quirúrgicamente por el especialista de Cirugía oral y Maxilofacial en el Hospital Metropolitano durante el periodo 2015-2018. La información se clasifico en base al diagnóstico, la edad, el sexo y el tipo de procedimiento quirúrgico. La prevalencia de patología de origen no traumático corresponde al 79,18\% y la de etiología traumática $20,82 \%$. Siendo el de mayor prevalencia dentro de la patología no traumática las anomalías dentofaciales, seguidas por infecciones de origen odontogénico, fisuras labio palatinas y patología oral.

La mayor prevalencia de cirugías realizadas durante el periodo 2015-2018 por el especialista Cirujano Oral y Maxilofacial en el Hospital Metropolitano corresponden a cirugías de etiología no traumática y dentro de este grupo la de mayor frecuencia es la cirugía ortognática para corrección de anomalías dentofaciales.

Palabras Claves: epidemiologia, tratamientos quirúrgicos, cirugía maxilofacial, estudio retrospectivo.

\section{Abstract}

Oral and Maxillofacial surgery is a surgical medical specialty. It deals with the diagnosis and treatment of facial trauma, facial abnormalities, lip cleft palate, infections of odontogenic origin, pathological, oncological or neoplastic processes that involving the oral cavity, face and craniofacial territory. Also, it includes cervical organs and structures directly or indirectly related to them. That need conservative or surgical treatment. The objective of this study is to determine the etiology of surgical treatments that were performed in the Metropolitan Hospital and its prevalence during the period 2015-2018.

A descriptive, quantitative, retrospective and transversal study was carried out because it collects data in a determined period. The sample of the research was 245 patients who were treated and surgically treated by the specialist of Oral and Maxillofacial Surgery at the "Hospital Metropoliano" during the period 2015-2018. The information was classified based on the diagnosis, age, sex and type of surgi- 
cal procedure. The prevalence of pathology of non-traumatic origin corresponds to $79.18 \%$ and that of traumatic etiology $20.82 \%$. The dentofacial anomalies are the most prevalent in non-traumatic pathology, followed by infections of odontogenic origin, palatal cleft lip and oral pathology. The highest prevalence of surgeries performed during the period 2015-2018 by the specialist oral and maxillofacial surgeon at the "Hospital Metropoliano", corresponds to surgeries of non-traumatic etiology and within this group the most frequent is surgery orthognathic for correction of dentofacial anomalies

Key words: epidemiology, surgical treatments, maxillofacial surgery, retrospective study.

\section{Introducción}

La Cirugía Oral y Maxilofacial, es una especialidad medico quirúrgica que se ocupa del diagnóstico y tratamiento de traumatismos faciales como fracturas tipo Lefort I, II y III, orbito cigomáticas, naso-orbito-etmoidales, mandibulares, entre otras; deformidades faciales como hipoplasia maxilar, prognatismo mandibular, laterognasias; fisuras de la 0 a la 14 de Tessier y procesos patológicos, oncológicos o neoplásicos que involucran la cavidad bucal, cara y territorio craneofacial así como de los órganos y estructuras cervicales relacionadas directa o indirectamente con las mismas y que deben ser atendidos quirúrgicamente ${ }^{1,2,3}$.

La mayoría de las urgencias maxilofaciales corresponden a traumatismos, que implican una alta morbilidad, alteración estética y perdida de la función². Según Pérez y cols. En el 2015 el 56\% de las intervenciones quirúrgicas corresponden al trauma facial y $44 \%$ a otras etiopatogenias no traumáticas.

El manejo del trauma facial ha sido por años unos de los campos mejor manejados por los cirujanos maxilofaciales. Las estructuras del esqueleto facial están articuladas e interdigitadas en un complejo, por lo tal, la fractura de un hueso afecta a las estructuras vecinas 4 . La experiencia y conocimiento de la anatomía del territorio bucal y maxilofacial proveen las herramientas necesarias para el manejo conservador o quirúrgico (reducción y fijación de fracturas con material de osteosíntesis) de las diferentes fracturas mandibulares, maxilares, cigomático-maxilares, naso-orbito-etmoidales, frontales y de la órbita ${ }^{1}$

Las intervenciones quirúrgicas no traumáticas según Pérez y cols. (2015) corresponden a infecciones de origen odontogénico que afectan a cabeza y cuello en un $27.6 \%$, tumores $27.2 \%$, cirugía craneofacial $26.9 \%$, cirugía reconstructiva $4.9 \%$, disfunción de Articulación Temporo Mandibular 3.8\%, displasias $0.75 \%$, retiro de material de osteosíntesis $0.75 \%$ y otras lesiones $2.6 \%$.

Los tratamientos quirúrgicos incluyen diversos procedimientos relacionados con trastornos de la articulación temporo mandibular, infecciones maxilofaciales, ci- 
rugía ortognática, cirugía craneofacial, tratamiento de las anomalías congénitas y del desarrollo maxilofacial, patología oral y enfermedades de glándulas salivales ${ }^{3}$

El objetivo de este estudio fue determinar la prevalencia los tratamientos quirúrgicos según su etiología de origen traumático o no traumático que realiza el Cirujano Oral y Maxilofacial durante su práctica privada hospitalaria con el fin de conocer la preparación y habilidad adquirida para la identificación y resolución de las diferentes lesiones del área maxilofacial.

\section{Materiales y métodos}

Se realizó un estudio de tipo descriptivo, cuantitativo, retrospectivo y transversal. La muestra de la investigación fue de 245 pacientes 123 mujeres y 122 hombres que fueron atendidos e intervenidos quirúrgicamente por el especialista de Cirugía Oral y Maxilofacial en el Hospital Metropolitano durante el periodo 2015-2018. La información se clasifico en base al diagnóstico, la edad, el sexo y el tipo de procedimiento quirúrgico. usando el programa IBM-SPSS Statistics 2.3 y Microsoft Exel. Para las variables continuas (edad) se calculó la media con sus respectivas desviaciones estándar (DE); mientras que el resto de las variables categóricas y se presentan en porcentajes.

La información recolectada se clasificó en dos grupos patología de origen traumática y no traumática. A su vez la patología de origen traumática se clasifico de acuerdo al tipo de fractura en: Fractura mandibular, maxilar, dentoalveolar, de orbita, de complejo orbito cigomático maxilar, de arco cigomático, heridas de tejido blando y secuela de trauma facial. Y la patología de origen no traumática se clasifico de acuerdo a su etiología en anomalía dentofacial, fisura labio y/o paladar, infecciones de origen odontogénico, patología oral y maxilofacial, atrofia maxilo mandibular y otros / cirugía oral.

\section{Análisis de resultados}

Los datos recolectados fueron clasificados y procesados en tablas y graficas para su análisis. Se analizaron 245 historias clínicas de las cuales 123 son mujeres $50,2 \%$ y 122 hombres $49,8 \%$; con una media de edad de 30,39 años. El mayor rango de pacientes se encuentra entre los 12 y 24 años que corresponden al $28,16 \%$ y el menor rango corresponde a pacientes mayores de 84 años, esto representa el $2 \%$ de la muestra total. (Ver Gráfica 1).

Los datos fueron clasificados en dos grupos: de origen traumático (Grupo 1) y no traumático (Grupo 2) en la Tabla 1 y 2 se puede ver la prevalencia de la edad y sexo según su etiología Grupo 1 y Grupo 2.

En términos de población general sin agrupación el diagnóstico general más común es la Anomalía Dentofaciales con 59 casos que representan el $24.08 \%$ de los datos totales seguido del trauma facial con 51 casos, es decir, el $20.82 \%$ de los da- 
tos, el diagnóstico menos frecuente fue atrofia maxilar con 12 casos registrados que representan tan solo el $4.9 \%$ del total de datos recolectado. (Ver Gráfica 2) Se puedo observar que de los 245 pacientes 194 corresponden al Grupo 2 que es $79,2 \%$ y 51 al Grupo 1 que es el 20,8\%. En el Grupo se clasificaron de acuerdo con el tipo de fractura como se ve en la Tabla 3 siendo la de mayor prevalencia las fracturas de complejo orbito cigomático maxilar.

En el Grupo 2 se clasificaron según su etiología donde podemos observar que el mayor porcentaje de cirugía de origen no traumática corresponde a las Anomalías dentofaciales en un $30,41 \%$, seguido por el de las infecciones de origen odontogénico en un $23,19 \%$ y cirugía de fisuras labio palatinas en un $20,61 \%$. Siendo el menor porcentaje la cirugía de recontruccion para atrofias maxilares que corresponde al $6.81 \%$ del total del Grupo 2. (Ver Tabla 4).

Gráfica 1

\section{Prevalencia de edad}

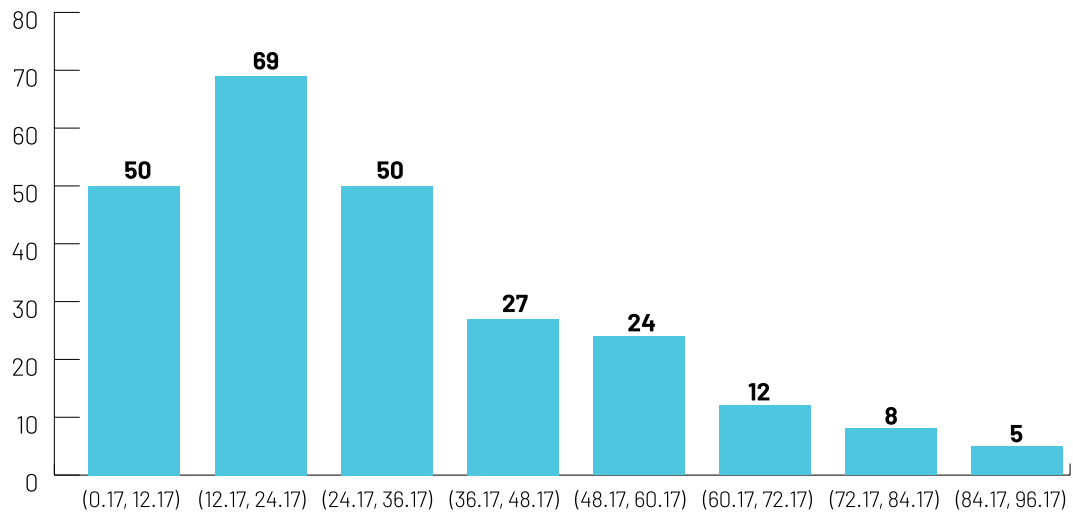

Tabla 1

\begin{tabular}{|c|c|c|c|c|c|c|c|c|}
\hline \multirow{3}{*}{ GRUPOS } & \multicolumn{4}{|c|}{ SEXO } & & & & \\
\hline & \multicolumn{2}{|c|}{ Femenino } & \multicolumn{2}{|c|}{ Masculino } & & & & \\
\hline & $\mathbf{N}$ & $\%$ & $\mathbf{N}$ & $\%$ & $\begin{array}{c}\text { Meedia } \\
\text { Edad }\end{array}$ & $\begin{array}{l}\text { Desv } \\
\text { Est } \\
\text { Edad }\end{array}$ & Total \% & $\begin{array}{c}\text { Total } \\
\text { general }\end{array}$ \\
\hline Grupo 1 & 17 & 6,94 & 34 & 13,88 & 29,31 & 17,75 & 20,82 & 51 \\
\hline Grupo 2 & 106 & 43,27 & 88 & 35,92 & 30,68 & 21,86 & 79,18 & 194 \\
\hline Total general & 123 & 50,20 & 122 & 49,80 & 30,39 & 21,03 & 100,0 & 245 \\
\hline
\end{tabular}


Tabla 2

\begin{tabular}{|c|c|c|c|c|c|c|c|c|}
\hline \multirow{3}{*}{ CÓDIGO } & \multicolumn{4}{|c|}{ SEXO } & \multirow[b]{3}{*}{$\begin{array}{l}\text { Media } \\
\text { Edad }\end{array}$} & \multirow[b]{3}{*}{$\begin{array}{l}\text { Desv } \\
\text { Est } \\
\text { Edad }\end{array}$} & \multirow[b]{3}{*}{ Total \% } & \multirow[b]{3}{*}{$\begin{array}{c}\text { Total } \\
\text { general }\end{array}$} \\
\hline & \multicolumn{2}{|c|}{ Femenino } & \multicolumn{2}{|c|}{ Masculino } & & & & \\
\hline & $\mathbf{N}$ & $\%$ & $n$ & $\%$ & & & & \\
\hline 1 & 17 & 6,94 & 34 & 13,8 & 29,31 & 17,75 & 20,82 & 51 \\
\hline 2 & 43 & 17,5 & 16 & 6,53 & 23,61 & 10,09 & 24,08 & 59 \\
\hline 3 & 13 & 5,31 & 27 & 11,0 & 8,67 & 9,95 & 16,33 & 40 \\
\hline 4 & 25 & 10,2 & 20 & 8,16 & 48,02 & 19,49 & 18,37 & 45 \\
\hline 5 & 11 & 4,49 & 10 & 4,08 & 36,81 & 24,14 & 8,57 & 21 \\
\hline 6 & 8 & 3,27 & 4 & 1,63 & 35,33 & 15,35 & 4,90 & 12 \\
\hline 7 & 6 & 2,45 & 11 & 4,49 & 45,12 & 26,80 & 6,94 & 17 \\
\hline $\begin{array}{c}\text { Total } \\
\text { general }\end{array}$ & 123 & 50,2 & 122 & 49,80 & 30,39 & 21,03 & 100,00 & 245 \\
\hline
\end{tabular}

Tabla 3

\begin{tabular}{c|l|c|c}
\hline \multicolumn{1}{c|}{ Código } & \multicolumn{1}{|c}{ Tipo de Fractura } & Frecuencia & $\%$ \\
\hline 1 & $\begin{array}{l}\text { FRACTURA DE COMPLEJO ORBITO CIGOMATICO } \\
\text { MAXILAR }\end{array}$ & 14 & 27,45 \\
\hline 2 & FRACTURA MANDIBULAR & 11 & 21,57 \\
\hline 3 & FRACTURA DE ORBITA & 9 & 17,65 \\
\hline 4 & SECUELA TRAUMA FACIAL & 7 & 13,73 \\
\hline 5 & FRACTURA DENTOALVEOLAR & 3 & 5,88 \\
\hline 6 & FRACTURA MAXILAR & 3 & 5,88 \\
\hline 7 & HERIDA TEJIDO BLANDO & 2 & 3,92 \\
\hline 8 & LUXACION DE MANDIBULA & 1 & 1,96 \\
\hline 9 & FRACTURAARCO CIGOMATICO & 1 & 1,96 \\
\hline & Total genEMal & 51 & 100,00
\end{tabular}


Tabla 4

\begin{tabular}{c|l|c|c}
\multicolumn{1}{c}{ Código Tipo patología } & Frecuencia & $\%$ \\
\hline 1 & ANOMALIAA DENTOFACIALES & 59 & 30,41 \\
\hline 2 & INFECCIONES ODONTOGÉNICAS & 45 & 23,19 \\
\hline 3 & FISURA LABIO / PALATINA & 40 & 20,61 \\
\hline 4 & PATOLOGÍA ORAL & 21 & 10,82 \\
\hline 5 & OTROS/ CIRUGIA ORAL & 17 & 8,76 \\
\hline 6 & ATROFIAMAXILAR & 12 & 6,18 \\
\hline & TOTAL GENERAL & 194 & 100,00
\end{tabular}

\section{Discusión}

Este estudio retrospectivo se realizó en el Hospital Metropolitano ubicado en la ciudad de Quito que brinda atención médica privada a la población general.

Existen pocos estudios que consideran los tratamientos quirúrgicos de etiología traumática, así como no traumática como: anomalía dentofaciales, patología, infecciones odontogénicas, fisuras labio palatinas, atrofia maxilo-mandibular, exodoncias y colocación de implantes dentales. La mayoría de estudias buscan la prevalencia una etiología específica y no así de los diferentes procedimientos quirúrgicos maxilofaciales.

Pérez y cols. (2015) en su estudio reportan una incidencia del $56 \%$ de cirugía de origen traumático en comparación del $44 \%$ cirugías de etiología no traumática mientras que Elorza y otros (2009) reportó una incidencia mayor de cirugía de origen no traumático 90,6 \% y 9,4\% corresponde a trauma facial. Se debe considerar que el estudio de Pérez y otros (2015) se realizó en un Hospital Público a diferencia del estudio de Elorza y otros (2009). que corresponde a un Hospital de practica Privada y de ahí deriva la diferencia del motivo de consulta, diagnóstico y tratamiento que se ejecutó ${ }^{2,5}$.

Nuestro estudio reportó una mayor incidencia de cirugía no traumática que representa el $79.18 \%$ del estudio mientras que la cirugía de etiología traumática fue del $20,8 \%$, que se asemeja más al estudio realizado por Elorza y cols. (2009) ya que ambos son Hospitales privados y difiere de estudios previos como el de Pérez y cols. (2015) donde la mayor incidencia corresponde a trauma facial.

Del total de pacientes atendidos en este estudio no se observó una predilección por el sexo pues el $50.2 \%$ corresponden a mujeres y $49,8 \%$ a hombres; similar al resultado de Elorza y cols. (2009) estadísticamente con 339 mujeres que corresponde al $52,3 \%$ y 309 hombres que corresponde al 47,6\%; sin embargo, Pérez y otros (2015) observó una mayor prevalencia en hombres $67,3 \%$ y del $32 ; 7 \%$ en mujeres dando una relación $2: 1$. 
Según Pérez y cols. (2015) la edad promedio del total de pacientes es de 38 años, así como también para Sandoval T. (2017) donde la edad promedio el total de pacientes es de 38 años y Elorza y otros (2009) reporta un rango de edad ente 21- 30 años. En nuestro estudio la edad promedio del total de los 245 pacientes estudiados es de 30,4 años donde el $28,18 \%$ tiene entre los 12 a 24 años. Esto indica que el rango de pacientes con patología maxilofacial se encuentra entre la tercera y cuarta década de la vida ${ }^{2,3,5}$.

El trauma maxilofacial es la primera causa de muerte y de baja productividad laboral entre la cuarta década de vida. Además, tiene una alta morbilidad por la pérdida de función, alteraciones estéticas y costo económico. Sandoval T. (2017). Como ya se mencionó la tasa de incidencia de nuestro estudio fue de $20,8 \%$ bajo en relación a trauma facial visto en la medicina de practica pública³.

Mijiti y cols. (2014) reportan una media de edad de 31,9 años en trauma facial Observando así que no existe una diferencia significativa con nuestro estudio donde se encontró una media de edad de 29,3 años, es decir que el trauma facial tiene mayor prevalencia en la tercera década de la vida ${ }^{6}$.

En cuanto a la distribución por el sexo y patología traumática según Pérez y cols. (2015) encontró que el $67,3 \%$ correspondía el sexo masculino y $32,7 \%$ a sexo femenino. dando una relación de 2:1. Bonavolonta y cols ( 2017) reporto también una incidencia mayor en hombres del $64,4 \%$ y del $35,6 \%$ en mujeres con una relación $1.8: 1$, nuestro resultado al igual que estudios anteriores también indica mayor prevalencia por el sexo masculino $66,6 \%$ y en sexo femenino de $33,3 \%$, con una relación de 2:1 1,2.

Las fracturas más comunes son las fracturas mandibulares; mientras que en el tercio medio las fracturas más comunes son las cigomáticas y maxilares. En estos casos el tratamiento de elección es la reducción abierta y fijación material de osteosíntesis. Naveen y cols. (2012). Algunos estudios reportan que la fractura más frecuente es la mandibular con una incidencia del $38,3 \%$ seguida por la fractura cigomática 27,2\%. Mijiti y cols. (2014) así Sandoval T. (2017) reporto una mayor incidencia en fracturas mandibulares del $68,3 \%$ seguido por fracturas del tercio medio facial en un $22,2 \%$. Sin embargo difiere de los resultados de Cabalag y cols. (2014) donde se mostró una mayor incidencia de fracturas de la órbita en un $36,63 \%$ seguido por fracturas maxilares en un $32,96 \%$, fracturas del cigoma en un $31,85 \%$ y fracturas mandibulares en un $16,94 \%$ 3,4,6,7.

En nuestro estudio se observó una prevalencia de fracturas de complejo orbito cigomático maxilar con una incidencia de $\%, 27,45 \%$ seguido por fracturas mandibular en un $21,57 \%$, fracturas de la órbita en un 17,65\% y el índice más bajo en fracturas aisladas de arco cigomático con un 1,96\%. Por lo tal en la mayoría de los estudios, así como el nuestro la incidencia de fracturas faciales es mayor en la mandíbula y cigoma.

De las cirugías de etiología no traumática en nuestro estudio la de mayor prevalencia corresponde a la cirugía de anomalías dentofaciales en un 30,41\%. Las anomalías dentofaciales son resultado de la interacción compleja de múltiples factores 
que influyen en el desarrollo y crecimiento; son así una de las etiologías de mayor incidencia en cirugía maxilofacial. Delgado y Villalpado (2005) como resultado de su estudio muestra una incidencia del 39\% de cirugías ortognáticas relacionadas a anomalías dentofaciales ${ }^{8}$.

En relación con la cirugía ortognática Cueto y cols. (2017) muestran en su estudio una predilección por el sexo femenino con $69,1 \%$ y sexo masculino de $30,9 \%$ al igual que nuestro estudio predilección por el sexo femenino en un $17,5 \%$ en comparación con sexo masculino en un 6,53\%; dando una relación de 2.7:1; así mismo en su estudio una edad promedio de 12,31 años, la misma que difiere en nuestro estudio donde la edad promedio corresponde a 23,6 años ${ }^{9}$.

Las infecciones odontogénicas, así como sus complicaciones tienen manifestaciones sistémicas y atentan contra la vida del paciente por ello es vital su diagnóstico temprano y tratamiento quirúrgico y/o hospitalario por lo que representa uno de los diagnósticos de etiología no traumática más comunes ${ }^{10}$.

Para Pérez y cols. (2015) las infecciones son la primera etiología de cirugía no traumática. Sandoval T. (2017) reportan una incidencia de 46,4\% de Infecciones odontogénicas mientras que Pérez y otros (2015) encontró una incidencia de del $27.6 \%$. En nuestro estudio ocupa el segundo lugar de incidencia de patología no traumática con un $23,19 \%$, sin predilección estadísticamente significativa por el sexo encontrándose una prevalencia de $10,2 \%$ en mujeres y $8,16 \%$ en hombres; y una media de edad de 48,02 años ${ }^{2,3}$.

Las fisuras labio palatinas son malformaciones congénitas de alta prevalencia, se presenta en 1 de cada 700 nacidos vivos, la misma que varía según la raza, etnia, origen geografía, sexo y condición socioeconómica ${ }^{11}$.

Pérez y cols. (2015) las agrupa como dismorfosis cráneo dentofaciales con una incidencia del $26,9 \%$, en nuestro estudio se observó una incidencia de $20,61 \%$ de fisuras labio platinas, así como sus secuelas y complicaciones, siendo la tercera causa de etiología no traumática de cirugía. El diagnostico de fisuras labio palatinas es más prevalente en hombres que en mujeres Biella y cols. (2014) observaron una prevalencia del $63,84 \%$ y $36,16 \%$ respectivamente; dando una relación de 1,8:1 similar a la relación analizada en nuestro estudio de 2,1: 1 con una incidencia de $11,0 \%$ en hombres y de $5,3 \%$ en mujeres ${ }^{2,12}$.

Las lesiones de la cavidad oral y maxilofacial agrupan un amplio conjunto de alteraciones que se localizan en los tejidos blandos y óseos; que se diferencian por su etiología, características clínicas e imagenológicas, pronostico y tratamiento ${ }^{13}$.

En nuestro estudio la patología oral fue agrupada en quistes, tumores, lesiones orales y patología de glándulas salivales con una incidencia del 10,8\%; correspondiendo a la cuarta etiología más común de etiología no traumática. Elorza y cols. (2009) reportaron una incidencia del 10\% de cirugía de tumores y según Pérez y cols. (2015) la patología oral tiene una prevalencia del 30, 55\% que incluye tumores, displasias y lesiones orales ${ }^{2,5}$. 
Para Fierro y cols. (2011) la edad media es de 54 años, sin predominio significativo por el sexo, con una tasa de incidencia en mujeres del $50,8 \%$ y en hombres del $49,2 \%$. Mientras que en nuestro estudio la edad media fue de 36,81 años que difiere con estudios previos donde es más frecuente en la quinta década del a vida, sin embargo, también muestra que no existe una prevalencia por el sexo; así se identificó una incidencia en hombres del $4,08 \%$ y en mujeres del $4,49 \%{ }^{14}$.

El tratamiento de elección para la atrofia maxilar es la cirugía de reconstrucción la misma que se realiza con diferentes tipos de injertos, y/o distracción osteogénica así mismo la cirugía reconstructiva está orientada a tratamientos oncológicos con el uso de colgajos óseos microvascularizados 15. En nuestro estudio la atrofia maxilar se presentó con la tasa de incidencia más baja de los procedimientos quirúrgico de origen no traumático con un $6,18 \%$ y una edad media de 35,33 años, más frecuente en mujeres con un $3,27 \%$ que en hombres $1,63 \%$.

Los procedimientos de cirugía oral como exodoncias, colocación de implantes dentales, regularización de rebordes que se realizaron bajo anestesia general por razones especiales como edad avanzada del paciente o alergia a los anestésicos locales. Elorza y cols. (2009) reportaron una prevalencia de $14 \%$ mientras que en nuestro estudio es de $8,76 \%$, con una edad media 45,12 años, presentándose mayo frecuencia en hombres $4,49 \%$ que en mujeres $2,45 \%$ es decir una relación 1.8:1 ${ }^{5}$.

La prevalencia está condicionada al tipo de Hospital público o privado. Como se demuestra en el estudio de Pérez y cols. (2015) en un hospital público la mayor prevalencia corresponde a fracturas faciales; lo que difiere del estudio de Elorza y otros (2009) en un hospital privado donde la mayor prevalencia es de cirugía ortognática asociada a anomalías dentofaciales ${ }^{2,5}$.

Los resultados de nuestra investigación coinciden con los de Elorza y cols. (2009) ya que ambos se realizaron en un hospital de practica privada; donde la mayor prevalencia corresponde a la etiología de anomalías dentofaciales las mismas que fueron tratadas con cirugía ortognática ${ }^{5}$.

Se asocia también la tasa de prevalencia con la gravedad de la etiología, por lo tal el trauma facial, las infecciones de origen odontogénico y patología que pone en riesgo la vida del paciente tienen una incidencia mayor.

Las cirugías electivas como es el caso de anomalías dentofaciales tienen una mayor incidencia en hospitales privados que públicos debido a lo limitados recursos físicos, técnicos y tiempo del hospital público que hace necesario priorizar patologías que comprometen la vida o función del paciente.

Los resultados obtenidos en este estudio permiten identificar a las anomalías dentofaciales como la etiología de mayor prevalencia, seguido por traumatismos faciales, infecciones de origen odontogénico, fisuras labio / palatinas y patología oral; lo que nos indica el enfoque y aprendizaje que requiere el médico tratante y postgradista para resolver las diferentes patologías que se presentan en la práctica privada y por lo tal conocer en que se requiere mayor especialización. 


\section{Conclusiones}

- Los tratamientos quirúrgicos realizados por el servicio de Cirugía Maxilofacial del Hospital Metropolitano fueron un total de 245 procedimientos de los cuales $194(79.18 \%)$ corresponde a lesiones de origen no traumático y $51(20,8 \%)$ a lesiones de etiología traumática.

- La etiología de mayor prevalencia en nuestro estudio es la anomalía dentofaciales que corresponden al 30, 41\% de las lesiones de etiología no traumática. Se considera a su tratamiento como una cirugía electiva, no urgente, que no llega a comprometer la vida del paciente. Por lo tal su prevalencia es mayor en los Hospitales Privadados.

- La cirugía de etiología traumática está asociada con lesiones que comprometen la vida o función del paciente por lo que su tratamiento es prioridad en los hospitales de practica pública. 


\section{Referencias bibliográfica}

1. Bonavolonta, P., Dell'aversana, G., Abbate, V., Vaira, L., Lo Faro, C., Petrocelli, M., \& Attanasi , F. The epidemiological analysis of maxillofacial fractures in Italy: The. Journal of Cranio-Maxillo-Facial Surgery. 2017; 45:1319-1326.

2. Pérez, H., Donoso, T., Mardones, M., \& Bravo, R. Epidemiología de Tratamientos Quirúrgicos Maxilofaciales en un Hospital Público en Santiago de Chile: Estudio Retrospectivo de 5 Años. Internacional Journal Odontostomatologia. 2015; 9(1): 37-41.

3. Sandoval T, R. D. Epidemiología de la patología quirúrgica que afecta al territorio maxilofacial, tratada bajo anestesia general en el Hospital de Urgencia Asistencia Pública entre 2014-2016. Revista Chilena de Cirugia.2017; 4(69): 289-296.

4. Naveen Shankar, A., Naveen Shankar, V., Hegde, N., \& Prasad, R. The pattern of the maxiIlofacial fractures e A multicentre retrospective study. Journal of Cranio-Maxillo-Facial Surgery.2017; 40: 675-679.

5. Elorza, C., Hincapié, E., Ramírez, P., \& Correa, P. Caracterización de pacientes tratados por Cirugía Maxilofacial en la Clínica CES 2005-2007. Rev.CES Odont. 2009; 22(1): 15-19.

6. Mijiti, A., Ling, W., Tuerdi, M., Maimaiti, A., Tuerxun, J., \& Tao, Y. Epidemiological analysis of maxillofacial fractures treated at a. Journal of Cranio-Maxillo-Facial Surgery.2014; 42: 227-233.

7. Cabalag, M., Wasiak, J., Andrew, N., Tang, J., Kirby, J., \& Morgan, D. Epidemiology and management of maxillofacial fractures in an Australian trauma centre. Journal of Plastic, Reconstructive \& Aesthetic Surgery. 2014; 67: 183-189.

8. Delgado, B., \& Villalpado, M. Incidencia de deformidades dentofaciales en un hospital de especialidades. Revista Médica del Instituto Mexicano.2005; 43(2): 155-159.

9. Cueto, A., Skog, F., Muñoz, M., Espinoza , S., \& Martinez, D. Prevalencia de Anomalías Dentomaxilares y necesidad de tratameinto en adolecentes. Internacional Journal of Odontostomatology.2017; 11(3): 333-338.

10. Flynn, T., Shanti, R., Levi, M., Andamo, A., Kraut, R., \& Trieger, N. Severe Oodntogenic Infections, Part 1; Prospective Report. Journal Oral Maxillofacial Surgery. 2006; 64: 1093-1103.

11. Karoon, A. Cleft palate repair and variations. 2009; 42(1): 102-109.

12. Biella, V., De Carvalho, C., da Silva, G., Costa, B., \& Ribero, M. Prevalence, Cause, and Location of Palatal Fistula in Operated Complete Unilateral Cleft Lip and Palate: Retrospective Study. The Cleft Palate-Craniofacial Journal.2014; 51(2):158-164.

13. Raposo, A., Monsalves, M., Aravena, P., \& Sanhueza, A. Prevalencia de Lesiones de la Mucosa Oral en el Hospital Hernán Henríquez Aravena de Temuco. Int. J. Morphol. 2014; 29(2): 622-627.

14. Fierro, C., Almendros, N., Berini , L., \& Gay Escoda, C. Prevalence of biopsied oral lesions in a Department of Oral Surgery 2007-2009. Journal Clin Exp Dent. 2011; 3(2): 73-77.

15. Cha, H.-S., Kim, J.-W., Hwang, J.-H., \& Ahn, K.-M. Frequency of bone graft in implant surgery. Cha et al. Maxillofacial Plastic and Reconstructive Surgery. 2016; 38(19): 2-4. 